\title{
CONFORMAL GROUP WITH TWO OBSERVER INDEPENDENT SCALES.
}

\author{
NICOLA ROSSANO BRUNO \\ Dipartimento di Fisica, Università di Roma ROMA TRE, and INFN Sez. Roma Tre, \\ Via Vasca Navale 84, 00146 Roma, Italy \\ Departamento de Física, Universidad de Burgos, Pza. Misael Bañuelos s.n., \\ 09001 Burgos, Spain
}

\begin{abstract}
The Poincaré sector of a recently deformed conformal algebra is proposed to describe, after the identification of the deformation parameter with the Planck length, the symmetries of a new relativistic theory with two observer-independent scales (or DSR theory). Also a new non-commutative space-time is proposed. It is found that momentum space exhibits the same features of the DSR proposals preserving Lorentz invariance in a deformed way. The space-time sector is a generalization of the well known non-commutative $\kappa$-Minkowski space-time which however does not preserve Lorentz invariance, not even in the deformed sense. It is shown that this behavior could be expected in some attempts to construct DSR theories starting from the Poincaré sector of a deformed symmetry larger than Poincaré symmetry, unless one takes a variable Planck length. It is also shown that the formalism can be useful in analyzing the role of quantum deformations in the "AdS-CFT correspondence".
\end{abstract}

One of the most studied applications of quantum groups in physics is in the description of deformed spacetime symmetries that generalize classical Poincaré kinematics beyond the Lie-algebra level. Well-known examples are the $\kappa$-Poincaré 1 and the quantum null-plane (or light-cone) Poincaré 2 algebras. The deformation parameter has been interpreted as a fundamental scale which may be related with the Planck length. In fact, these results can be seen as different attempts to develop new approaches to physics at the Planck scale 3 . It has been also recently conjectured that the $\kappa$-Poincaré algebra may provide the basis for a so-called doubly special relativity (DSR) 415617 in which the deformation parameter/Planck length is viewed as an observer-independent length scale completely analogous to the familiar observer-independent velocity scale $c$, in such a manner that (deformed) Lorentz invariance is preserved 8910 . In this talk I want to review briefly some related results which have been presented in greater detail in 11, 12. These results provide an analysis of the Poincaré sector of a manageable deformation of $s o(4,2)$ introduced in [13] together with its dual in order to extract some physical implications on the associated non-commutative Minkowskian spacetime.

If $\left\{J_{i}, P_{\mu}=\left(P_{0}, \mathbf{P}\right), K_{i}, D\right\}$ denote the generators of rotations, time and space translations, boosts and dilations, the non-vanishing deformed commutation rules 
of $U_{\tau}(\mathcal{W} P)$ are given by 13 :

$$
\begin{array}{lll}
{\left[J_{i}, J_{j}\right]=\mathrm{i} \varepsilon_{i j k} J_{k}} & {\left[J_{i}, K_{j}\right]=\mathrm{i} \varepsilon_{i j k} K_{k}} & {\left[J_{i}, P_{j}\right]=\mathrm{i} \varepsilon_{i j k} P_{k}} \\
{\left[K_{i}, K_{j}\right]=-\mathrm{i} \varepsilon_{i j k} J_{k}} & {\left[K_{i}, P_{0}\right]=\mathrm{i} \mathrm{e}^{-\tau P_{0}} P_{i}} & {\left[D, P_{i}\right]=\mathrm{i} P_{i}} \\
{\left[K_{i}, P_{i}\right]=\mathrm{i} \frac{\mathrm{e}^{\tau P_{0}}-1}{\tau}} & {\left[D, P_{0}\right]=\mathrm{i} \frac{1-\mathrm{e}^{-\tau P_{0}}}{\tau}} &
\end{array}
$$

where hereafter we assume $\hbar=c=1$, sum over repeated indices, Latin indices $i, j, k=1,2,3$, while Greek indices $\mu, \nu=0,1,2,3$ and $\tau$ is a real deformation parameter. By means of familiar techniques developed for the study of DSR theories, finite boost transformations were obtained in [1] as well as a detailed analysis of the range of the boost parameter. It was found that the range of the rapidity depends on the sign of the deformation parameter and, in general, the behavior of the rapidity, energy and momentum differs from DSR theories based on $\kappa$-Poincaré. For positive deformation parameter, the situation is rather similar to the undeformed case, while for negative deformation parameter, the range of the rapidity is restricted between two values which are asymptotes for the energy. These values determine a maximum momentum that depends not only on the deformation parameter (as in the DSR of [4, 8) but also on the deformed mass of the particle. Furthermore two proposals for position and velocity operators were presented: one of them provides a variable speed of light for massless particles, while the other one gives rise to a fixed speed of light and also introduces a new type of generalized uncertainty principle.

From a dual quantum group perspective, when the quantum spacetime coordinates $\hat{x}^{\mu}$ conjugate to the $\kappa$-Poincaré momentum-space $P_{\mu}$ (translations) are considered, the non-commutative $\kappa$-Minkowski spacetime arises 14151161718. More general non-commutative Minkowskian spacetimes can be described by means of the following Lie algebra commutation rules 19 .

$$
\left[\hat{x}^{\mu}, \hat{x}^{\nu}\right]=\frac{1}{\kappa}\left(a^{\mu} \hat{x}^{\nu}-a^{\nu} \hat{x}^{\mu}\right),
$$

where $a^{\mu}$ is a constant four-vector in the Minkowskian space. In [12] the dual quantum group of (11) was analyzed making use of the quantum $\mathcal{R}$-matrix (the well known FRT approach 20, and giving rise to the new non-commutative spacetime

$$
\left[\hat{x}^{\mu}, \hat{x}^{\nu}\right]=\tau\left(\hat{\Lambda}_{0}^{\nu}(\hat{\xi}) \hat{x}^{\mu}-\hat{\Lambda}_{0}^{\mu}(\hat{\xi}) \hat{x}^{\nu}\right)
$$

which can be seen as a generalization of (2) through $a^{\mu} \rightarrow \hat{\Lambda}_{0}^{\mu}(\hat{\xi})$. Since $\hat{\Lambda}_{0}^{\mu}$ (which are formal quantum Lorentz entries) only depend on the quantum boost parameters and the quantum rotation coordinates $\hat{\theta}^{i}$ do not play any role in the spacetime non-commutativity, the isotropy of the space is thus preserved. The relations (3) show that different observers in relative motion with respect to quantum group transformations have a different perception of the spacetime non-commutativity, i.e., the equivalence of all the inertial frames is lost. Nevertheless, in this context, covariance under quantum group transformations is ensured by construction. 
We also stress that if the following new space variables $\hat{X}^{i}$ in the $(3+1) \mathrm{D}$ spacetime (3) are considered

$$
\hat{x}^{0} \rightarrow \hat{x}^{0}, \quad \hat{x}^{i} \rightarrow \hat{X}^{i}=\hat{x}^{i} \hat{\Lambda}_{0}^{0}-\hat{x}^{0} \hat{\Lambda}_{0}^{i},
$$

the transformed commutation rules for the quantum spacetime are given by

$$
\left[\hat{X}^{i}, \hat{x}^{0}\right]=\tau \hat{\Lambda}_{0}^{0}(\hat{\xi}) \hat{X}^{i}, \quad\left[\hat{X}^{i}, \hat{X}^{j}\right]=0,
$$

which, in turn, can be interpreted as a generalization of the $\kappa$-Minkowski space with a "variable" Planck length $\tau^{\prime}=\tau \hat{\Lambda}_{0}^{0}(\hat{\xi})$ that does depend on all the quantum boost parameters (in the $(1+1) \mathrm{D}$ case, this yields $\tau^{\prime}=\tau \cosh \hat{\xi}$ ). This result is a direct consequence of imposing a larger quantum group symmetry than Poincaré.

In closing, I want to stress that Ref. 12 also presents two maps that can be used to express the same quantum deformation of $s o(4,2)$ within two physically different frameworks: $U_{\tau}\left(\mathcal{C} M^{3+1}\right) \leftrightarrow U_{\tau}\left(A d S^{4+1}\right)$. In fact, such a quantum group relationship might further be applied in order to analyze the role that quantum deformations of $s o(4,2)$ could play in relation with the "AdS-CFT correspondence" that relates local QFT on $A d S^{(d-1)+1}$ with a conformal QFT on the (compactified) Minkowskian spacetime $\mathcal{C} M^{(d-2)+1}$.

\section{References}

1. J. Lukierski, A. Nowicki, H. Ruegg, V.N. Tolstoy, Phys. Lett. B 264 (1991) 331; S. Giller, P. Kosinski, J. Kunz, M. Majewski, P. Maslanka, Phys. Lett. B 286(1992)57; J. Lukierski, H. Ruegg, A. Nowicky, Phys Lett. B 293 (1992) 344.

2. A. Ballesteros, F.J. Herranz, M.A. del Olmo, M. Santander, Phys. Lett. B 351(1995)137; A. Ballesteros, F.J. Herranz, C.M. Pereña, Phys. Lett. B 391 (1997) 71.

3. S. Majid, Class. Quantum Grav. 51587 (1988).

4. G. Amelino-Camelia, Phys. Lett. B 510 (2001) 255; Int. J. Mod. Phys. D 11 (2002) 35.

5. J. Magueijo, L. Smolin, Phys. Rev. Lett. 88 (2002) 190403.

6. G. Amelino-Camelia, Int. J. Mod. Phys. D 11 (2002) 1643; Nature 418 (2002) 34.

7. J. Kowalski-Glikman, S. Nowak, Phys. Lett. B 539 (2002) 126.

8. N.R. Bruno, G. Amelino-Camelia, J. Kowalski-Glikman, Phys. Lett. B 522 (2001) 133.

9. N.R. Bruno, Phys. Lett. B 547 (2002) 109.

10. J. Lukierski, A. Nowicki, Int. J. Mod. Phys. A 18 (2003) 7.

11. A. Ballesteros, N.R. Bruno, F.J. Herranz, J. Phys. A 36 (2003) 10493.

12. A. Ballesteros, N.R. Bruno, F.J. Herranz, Phys. Lett B 574 (2003) 276.

13. F.J. Herranz, Phys. Lett. B 543 (2002) 89.

14. P. Maslanka, J. Phys. A 26 (1993) L1251.

15. S. Majid, H. Ruegg, Phys. Lett. B 334 (1994) 348.

16. S. Zakrzewski, J. Phys. A 27 (1994) 2075.

17. J. Lukierski, H. Ruegg, Phys. Lett. B 329 (1994) 189.

18. A. Agostini, G. Amelino-Camelia, F. D'Andrea, hep-th/0306013

19. J. Lukierski,V.D. Lyakhovsky, M. Mozrzymas, Phys. Lett. B 538 (2002) 375.

20. L.D. Faddeev, N.Y. Reshetikhin, L.A. Takhtajan, Lengingrad Math. J. 1 (1990) 193. 